\title{
Elektroenergie und Brennstofielemente
}

\author{
Von Prof. Dr. KURT SCHWABE
}

(Abhandlungen der Sächsischen Akademie der Wissenschaften zu Leipzig, mathematisch-naturwissenschaftliche Klasse, Band 49, Heft 2)

1966. 19 Seiten - 13 Abbildungen -8 Tabellen $-4^{\circ}-4,20 \mathrm{M}$

Der Verfasser gibt einen Überblick über die allgemeine Bedeutung, den Forschungsstand und die Perspektive der Brennstoffelemente (BE) im Zusammenhang mit dem ständig wachsenden Elektroenergiebedarf in der Welt.

Anhand von Beispielen aus der Praxis erläutert er die Vorteile und die verschiedenen Einsatzmöglichkeiten, z. B. im Betrieb strippenloser elektrischer Geräte oder langsamer Fahrzeuge. Dabei wird auch auf die Anwendbarkeit von Brennstoffelementen zur Energiespeicherung und insbesondere zur Industrialisierung von jüngeren Nationalstaaten hingewiesen.

Der Autor berichtet über seine eigene Forschungs- und Entwicklungsarbeit und berücksichtigt dabei besonders die für die DDR charakteristischen Gesichtspunkte. Es gelang ihm, neben neuen Vorstellungen zur Kinetik der Elektrodenprozesse mit Hilfe thermischer und elektrochemischer Verfahren eine leistungsfähige $\mathrm{C}_{2}$-Kohleelektrode zu entwickeln, die z. B. als Ersatz von $\mathrm{C}_{2}$-liefernden Importrohstoffen in galvanischen Elementen ebenso geeignet sind, wie für die energieeinsparende Ausnutzung großtechnischer Oxydationsprozesse mittels Brennstoffelementen. Mit Nachdruck verweist der Autor auf die Tatsache, daß ein so modernes Forschungsunternehmen heute nur in Zusammenarbeit zwischen Ingenieuren und Wissenschaftlern der verschiedensten Bereiche mit Erfolg durchgeführt werden kann.

\section{Bestellungen durch eine Buchhandlung erbeten}

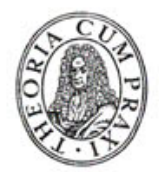

\section{A K A D E M I E - V E R L A G - B E R L I N}

\section{BEZUGS MÖGLICHKEITEN}

Sämtliche Veröffentlichungen unseres Verlages sind durch jede Buchhandlung im In- und Ausland zu beziehen. Falls keine Bezugsmöglichkeit vorhanden ist, wende man sich

in der Deutschen Demokratischen Republik

an den AKADEMIE-VERLAG, GmbH, 108 Berlin, Leipziger Straße 3-4

in der Deutschen Bundesrepublik

an KUNST UND WISSEN, Erich Bieber, 7 Stuttgart 1, Wilhelmstraße 4-6

in Österreich

an den GLOBUS-Buchvertrieb, Wien I, Salzgries 16

in Nord- und Südamerika

an Gordon and Breach Science Publishers, Inc., 150 Fifth Avenue, New York, N. Y. 10011 U.S.A.

bei Wohnsitz im übrigen nichtsozialistischen Ausland

an den Deutschen Buch-Export und -Import, GmbH, 701 Leipzig, Leninstraße 16.

Im sozialistischen Ausland können Bestellungen über die Buchhandlungen für fremdsprachige Literatur bzw. den zuständigen Postzeitungsvertrieb erfolgen.

Auf Wunsch sendet der AKADEMIE-VERLAG Interessenten bei Bekanntgabe der Anschrift und Fachgebiete unverbindlich Informationen über lieferbare und kommende Veröffentlichun gen und gibt auch Bezugsquellen im In- und Ausland bekannt. 